\title{
Réseau sécurisé des médecins suisses
}

La FMH a le plaisir de vous annoncer l'ouverture, dès le $1^{\text {er }}$ décembre 2000, de son bureau HIN Suisse romande (Health-Info-Net) à Lausanne. Afin d'être à l'écoute de ses membres et de leur offrir un service de proximité, la FMH a passé un contrat de partenariat avec Nice Computing. Cette action est la suite d'un processus qui a débuté en 1996 par la fondation de HIN SA, suivie de la décision prise en juin 2000 par la Chambre médicale suisse d'inclure l'abonnement de base à HIN dans la cotisation centrale à la FMH, à tout le moins pendant la période de démarrage.

Dès le 4 janvier 2001 l'Infoline HIN, le 0848830 741, sera prise en charge par HIN Suisse romande.

Tous les médecins de la FMH peuvent disposer de communications sécurisées sur Internet. Tous les utilisateurs suisses romands de HIN ont désormais un interlocuteur direct offrant support et assistance téléphonique (hotline) pour l'installation des systèmes de sécurité.

HIN Suisse romande offre un service rapide et soigné et développe des prestations à forte valeur ajoutée pour tous les intervenants dans le secteur de la santé.

HIN Suisse romande dispose d'une équipe compétente au service des acteurs de la santé. Les collaborateurs de Nice Computing ont été étroitement associés aux développements du consortium européen "SynEx - Synergy on the Extranet» centré sur la communication des données médicales au niveau européen. Les outils mis en place dans le cadre de ce projet sont le fruit d'une étroite coopération entre les HUG (Hôpitaux universitaires de Genève), la FMH, le HIN, l’AMG (Association des médecins du canton de Genève) et l'HEGP (Hôpital européen Georges Pompidou à Paris).

En plus de la sécurisation des communications, HIN Suisse romande offre des cours de formation, des développements sur serveurs sécurisés ainsi que d'autres services plus spécifiques.

La question du maillon faible de la communication, la sécurité, a pu ainsi être aujourd'hui résolue aisément et à moindre coût pour tous les médecins, les hôpitaux, les cliniques ainsi que pour les autres intervenants sur le terrain de la santé.

Fédération des médecins suisses (FMH)

Le secrétaire général:

François-X. Deschenaux

Président du conseil d'administration de HIN SA

Corrigendum

\section{Salaire des assistantes médicales pour 2001 et recommandations de salaires}

(Bulletin des médecins suisses no 28/2000 du 29 novembre 2000)

L’Office fédéral de la statistique a corrigé l'indice suisse des prix à la consommation: le renchérissement au mois de septembre 2000 est de 1,4\% et de 1,3\% en octobre. 